\title{
Bearing-Only Control Laws For Balanced Circular Formations of Ground Robots
}

\author{
Nima Moshtagh, Nathan Michael, Ali Jadbabaie, Kostas Daniilidis \\ GRASP Laboratory \\ University of Pennsylvania, Philadelphia, PA 19104 \\ Email: \{nima, nmichael, jadbabai, kostas\}@grasp.upenn.edu
}

\begin{abstract}
For a group of constant-speed ground robots, a simple control law is designed to stabilize the motion of the group into a balanced circular formation using a consensus approach. It is shown that the measurements of the bearing angles between the robots are sufficient for reaching a balanced circular formation. We consider two different scenarios that the connectivity graph of the system is either a complete graph or a ring. Collision avoidance capabilities are added to the team members and the effectiveness of the control laws are demonstrated on a group of mobile robots.
\end{abstract}

\section{INTRODUCTION}

Inspired by the social aggregation phenomena in birds and fish [1]-[3], researchers in robotics and control theory have been developing tools, methods and algorithms for distributed motion coordination of multi-vehicle systems. Two main collective motions that are observed in nature are parallel motion and circular motion [4]. One can interpret stabilizing the circular formation as an example of activity consensus, that is, individuals are "moving around" together. Stabilizing the parallel formation is another form of activity consensus in which individuals "move off" together [5].

The circular formation is a circular relative equilibrium in which all the agents travel around the same circle. This kind of behavior is observed in fish schooling, a well studied topic in ecology and evolutionary biology [3]. The balanced formation is an interesting family of equilibrium states where the agents are evenly spaced on a circular trajectory, and the geometric center of the agents is fixed. At the equilibrium, the relative headings and the relative distances of the agents determine the shape of the formation [6].

The primary contribution of this work is the presentation of a simple control law for achieving a balanced circular formation that only requires visual sensing such as bearing angles, i.e., the input is in terms of quantities that do not require communication among nearest neighbors. In contrast with the work of Paley et al. [5], Sepulchre et al. [6], and Moshtagh et al. [7], where it is assumed that each agent has access to the values of its neighbors' positions and velocities, we design distributed control laws that use only visual clues from nearest neighbors to achieve motion coordination.

In [8]-[10] circular formations of a multi-vehicle system under cyclic pursuit is studied. The proposed strategy is distributed and relatively simple because each agent needs to measure the relative information from only one other agent. It is also shown that the formation equilibria of the multi-agent system are generalized polygons. In contrast to [8] our control law is a nonlinear function of the bearing angles and as a result our system converges to a different set of stable equilibria.

Verification of the theory through multi-robot experiments demonstrated the effectiveness of the bearing-only control law to achieve the desired formation. Of course in reality any formation control requires collision avoidance, and indeed collision avoidance cannot be done without range. In order to improve the experimental results, we provided inter-agent collision avoidance properties to the team members. What we show in this work is that the two tasks of formation-keeping and collision-avoidance can be done with decoupled additive terms in the control law, where the terms for keeping circular formation depends only on the bearing parameters.

The outline of the paper is as follows. First we provide some background information on graph theory and polygons that we are going to use throughout the paper. In Section III we derive the bearing-only controller that stabilizes a group of mobile agents into a balanced circular formation. In Section IV collision avoidance capabilities are added to the control laws. The derived controllers are tested on real robots and the experimental results are presented in Section V.

\section{BACKGROUND}

In this section we briefly review a number of important concepts that we use in this paper.

\section{A. Graph Theory}

An (undirected) graph $\mathbb{G}$ consists of a vertex set, $\mathcal{V}$, and an edge set $\mathcal{E}$, where an edge is an unordered pair of distinct vertices in $\mathbb{G}$. If $x, y \in \mathcal{V}$, and $(x, y) \in \mathcal{E}$, then $x$ and $y$ are said to be adjacent, or neighbors and we denote this by writing $x \sim y$. The number of neighbors of each vertex is its valence. A path of length $r$ from vertex $x$ to vertex $y$ is a sequence of $r+1$ distinct vertices starting with $x$ and ending with $y$ such that consecutive vertices are adjacent. If there is a path between any two vertices of a graph $\mathbb{G}$, then $\mathbb{G}$ is said to be connected.

The adjacency matrix $A(\mathbb{G})=\left[a_{i j}\right]$ of an (undirected) graph $\mathbb{G}$ is a symmetric matrix with rows and columns indexed by the vertices of $\mathbb{G}$, such that $a_{i j}=1$ if vertex $i$ and vertex $j$ are neighbors and $a_{i j}=0$, otherwise. We also assume that $a_{i i}=0$ for all $i$. The valence matrix, $D(\mathbb{G})$, of a graph $\mathbb{G}$ 
is a diagonal matrix with rows and columns indexed by $\mathcal{V}$, in which the $(i, i)$-entry is the valence of vertex $i$.

The symmetric singular matrix defined as:

$$
L(\mathbb{G})=D(\mathbb{G})-A(\mathbb{G})
$$

is called the Laplacian of $\mathbb{G}$. The Laplacian matrix captures many topological properties of the graph. The Laplacian $L$ is a positive semidefinite M-matrix (a matrix whose off-diagonal entries are all nonpositive) and the algebraic multiplicity of its zero eigenvalue (i.e., the dimension of its kernel) is equal to the number of connected components in the graph. The $n$ dimensional eigenvector associated with the zero eigenvalue is the vector of ones, $\mathbf{1}_{n}=[1, \ldots, 1]^{T}$.

Given an orientation of the edges of a graph, we can define the incidence matrix of the graph to be a matrix $B$ with rows indexed by vertices and columns indexed by edges with entries of 1 representing the source of a directed edge and -1 representing the sink. The Laplacian matrix $L(\mathbb{G})$ of graph $\mathbb{G}$ is represented in terms of its incidence matrix as $L=B B^{T}$ independent of the orientation of the edges.

\section{B. Regular Polygons}

Let $d<n$ be a positive integer and define $p=n / d$. Let $y_{1}$ be a point on the unit circle. Let $R_{\alpha}$ be a clockwise rotation by the angle $\alpha=2 \pi / p$. The generalized regular polygon $\{p\}$ is given by the points $y_{i+1}=R_{\alpha} y_{i}$, and edges between points $i$ and $i+1$ [11].

When $d=1$ the polygon $\{p\}$ is called an ordinary regular polygon and its edges do not intersect. If $d>1$ and $n$ and $d$ are coprime, then the edges intersect and the polygon is a star. If $n$ and $d$ have a common factor $l>1$, then the polygon consists of $l$ traversals of the same polygon with $\{n / l\}$ vertices and edges. If $d=n$ the polygon $\{n / n\}$ corresponds to all points at the same location. If $d=n / 2$ (with $n$ even), then the polygon consists of two end points and a line between them, with points corresponding to an even index on one end and points corresponding to an odd index on the other.

\section{Kronecker Product}

The Kronecker product, denoted by $\otimes$, is an operation on two matrices of arbitrary size resulting in a block matrix. If $A$ is an $m \times n$ matrix and $B$ is a $p \times q$ matrix, then the Kronecker product $A \otimes B$ is a $m p \times n q$ block matrix. If $A, B, C$ and $D$ are matrices of such size that one can form the matrix products $A C$ and $B D$, then $(A \otimes B)(C \otimes D)=A C \otimes B D$. This is called the mixed-product property. Also the following property holds $(A \otimes B)^{T}=A^{T} \otimes B^{T}$.

\section{Circular Formations of Planar Robots}

Consider a group of $n$ unit-speed planar agents. Each agent is capable of sensing information from its neighbors. The neighborhood set of agent $i, \mathcal{N}_{i}$, is the set of agents that can be "seen" by agent $i$. The precise meaning of "seeing" will be cleared later. The size of the neighborhood depends on the characteristics of the sensors. The neighboring relationship between agents can be conveniently described by a graph.
Definition 3.1 (Connectivity Graph): The

connectivity graph $\mathbb{G}=\{\mathcal{V}, \mathcal{E}\}$ is a graph consisting of:

- a set of vertices $\mathcal{V}$ indexed by the set of mobile agents;

- a set of edges $\mathcal{E}=\{(i, j) \mid i, j \in \mathcal{V}$, and $i \sim j\}$;

The edge set $\mathcal{E}$ represents the links among the agents, and the neighborhood of agent $i$ is defined by

$$
\mathcal{N}_{i} \doteq\{j \mid i \sim j\} \subseteq\{1, \ldots, n\} \backslash\{i\} .
$$

A circular formation is a circular relative equilibrium in which all the agents travel around the same circle. At the equilibrium, the relative headings and the relative distances of the agents determine the shape of the formation. We are interested in balanced circular formations as defined by:

Definition 3.2 (Balanced Circular Formation): The set of equilibrium states where the agents are evenly spaced on a circular trajectory, and the geometric center of the agents is fixed is called the balanced circular formation.

\section{A. Kinematic Model for Mobile Robots}

Let $\mathbf{r}_{i}$ represent the position of agent $i$, and $\mathbf{v}_{i}$ be its velocity vector. The dynamics of each unit-speed agent is given by:

$$
\begin{aligned}
\dot{\mathbf{r}}_{i} & =\mathbf{v}_{i} \\
\dot{\mathbf{v}}_{i} & =\omega_{i} \mathbf{v}_{i}^{\perp} \\
\dot{\mathbf{v}}_{i}^{\perp} & =-\omega_{i} \mathbf{v}_{i}
\end{aligned}
$$

where $\mathbf{v}_{i}^{\perp}$ is the unit vector perpendicular to the velocity vector $\mathbf{v}_{i}$. The orthogonal pair $\left\{\mathbf{v}_{i}, \mathbf{v}_{i}^{\perp}\right\}$ forms a body frame for agent $i$ (See Figure 1). We represent the stack vector of all the velocities by $\mathbf{v}=\left[\mathbf{v}_{1}^{T}, \ldots, \mathbf{v}_{n}^{T}\right]^{T} \in \mathbb{R}^{2 n \times 1}$.

The control input for each agent is the angular velocity $\omega_{i}$. Since it is assumed that the agents move with constant unit speed, the force applied to each agent must be perpendicular to its velocity vector, i.e., the force on each agent is a gyroscopic force, and it does not change its speed (and hence its kinetic energy). Thus, $\omega_{i}$ serves as a steering control [12] for each agent. In the following we design a distributed control law for achieving a balanced formation.

Let $\mathbf{c}_{i}$ represent the position of the center of the $i$-th circle with radius $1 / \omega_{o}$, as shown in Figure 1 , thus

$$
\mathbf{c}_{i}=\mathbf{r}_{i}+\left(1 / \omega_{o}\right) \mathbf{v}_{i}^{\perp} .
$$

The shape controls for driving agents to a circular formation depend on the shape variables $\mathbf{v}_{i j}=\mathbf{v}_{j}-\mathbf{v}_{i}$ and $\mathbf{r}_{i j}=$ $\mathbf{r}_{j}-\mathbf{r}_{i}$. The relative equilibria of the balanced formation are characterized by $\sum_{i=1}^{n} \mathbf{v}_{i}=0$, and $\mathbf{c}_{i}=\mathbf{c}_{o} \in \mathbb{R}^{2}$ for all $i \in\{1, \ldots, n\}$, where $\mathbf{c}_{o}$ is the fixed geometric center of the agents.

The control input for each agent has two components:

$$
\omega_{i}=\omega_{o}+u_{i}
$$

The constant angular velocity $\omega_{o}$ takes the agents into a circular motion, and $u_{i}$ puts the agents into a balanced formation. In 


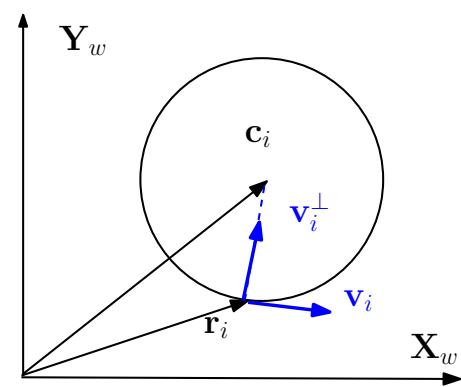

Fig. 1. Center of the circular trajectory is defined as $\mathbf{c}_{i}=\mathbf{r}_{i}+\left(1 / \omega_{0}\right) \mathbf{v}_{i}^{\perp}$.

order to design $u_{i}$ we express the system in a rotating frame, which greatly simplifies the analysis. By a change of variable

$$
\mathbf{z}_{i}=\omega_{o}\left(\mathbf{r}_{i}-\mathbf{c}_{i}\right)=-\mathbf{v}_{i}^{\perp}
$$

the problem reduces to balancing the agents on a unit circle as shown in Figure 2. The new coordinate system is rotating with angular velocity $\omega_{o}$. The dynamics in the rotating frame is given by

$$
\begin{aligned}
\dot{\mathbf{z}}_{i} & =\mathbf{v}_{i} u_{i} \\
\dot{\mathbf{v}}_{i} & =-\mathbf{z}_{i} u_{i}, \quad i=1, \ldots, n
\end{aligned}
$$

The new position vector $\mathbf{z}_{i}$ is a unit vector, however its speed $\left|\dot{\mathbf{z}}_{i}\right|$ is not constant anymore, and it is proportional to $u_{i}$, which goes to zero as the group reaches a balanced formation.

Let us define $\mathbf{z}_{i j}=\mathbf{z}_{j}-\mathbf{z}_{i}$ and $\mathbf{q}_{i j}=\mathbf{z}_{i j} /\left|\mathbf{z}_{i j}\right|$ as the unit vector along the relative position vector $\mathbf{z}_{i j}$. We note that at the balanced equilibrium the velocity of each agent must be perpendicular to $\overline{\mathbf{q}}_{i}=\sum_{j \in \mathcal{N}_{i}} \mathbf{q}_{i j}$, which is a vector along the average of the relative position vectors that are incident to agent $i$. Thus, the quantity $<\mathbf{v}_{i}, \overline{\mathbf{q}}_{i}>$ vanishes at the balanced equilibrium. Hence we propose the following control law for the balanced formation:

$$
u_{i}=-\kappa<\mathbf{v}_{i}, \overline{\mathbf{q}}_{i}>=-\kappa \sum_{j \in \mathcal{N}_{i}}<\mathbf{v}_{i}, \mathbf{q}_{i j}>, \kappa>0 .
$$

\section{B. Complete-Graph Topology}

Suppose the underlying connectivity graph is a complete graph. We have the following theorem for reaching the balanced circular formation in a group of mobile planar agents with a complete-graph connectivity.

Theorem 3.3: Consider a system of $n$ agents with kinematics (2). Given a complete connectivity graph $\mathbb{G}$, and applying control law (3), the n-agent system (almost) globally asymptotically converges to a balanced circular formation as defined in Definition 3.2.

Proof: Let us define vector $\mathbf{p}$ that points towards the geometric center of the group:

$$
\mathbf{p}=\frac{1}{n} \sum_{i=1}^{n} \mathbf{z}_{i}=\frac{1}{n} \mathbf{1}^{T} \mathbf{z}, \quad \mathbf{1}=\mathbf{1}_{n} \otimes I_{2} \in \mathbb{R}^{2 n \times 2} .
$$

The minimum $|\mathbf{p}|=0$ is reached when the position vectors $\mathbf{z}_{i}$ are in a balanced position (splay state); and the maximum

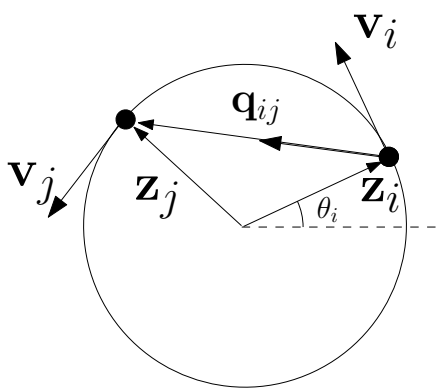

Fig. 2. By a change of coordinate $\mathbf{z}_{i}=\omega_{o}\left(\mathbf{r}_{i}-\mathbf{c}_{i}\right)=-\mathbf{v}_{i}^{\perp}$ the problem of generating circular motion in the plane reduces to the problem of balancing the agents on a circle.

$|\mathbf{p}|=1$ is reached when all the position vectors are aligned (state synchronized). Note that the balancing input (3) can be bounded above by a function of vector $\mathbf{p}$ :

$$
\begin{aligned}
u_{i} & =-\kappa \sum_{j \in \mathcal{N}_{i}}<\frac{\mathbf{z}_{i j}}{\left|\mathbf{z}_{i j}\right|}, \mathbf{v}_{i}>=-\kappa \sum_{j=1}^{n} \frac{1}{\left|\mathbf{z}_{i j}\right|}<\mathbf{z}_{j}, \mathbf{v}_{i}> \\
& \leq-\frac{\kappa}{|\mathbf{z}|_{\max }} \sum_{j=1}^{n}<\mathbf{z}_{j}, \mathbf{v}_{i}> \\
& \left.=-\frac{n \kappa}{|\mathbf{z}|_{\max }}<\mathbf{p}, \mathbf{v}_{i}\right\rangle
\end{aligned}
$$

where $|\mathbf{z}|_{\text {max }}=\max \left\{\left|\mathbf{z}_{i j}\right|,(i, j) \in \mathcal{E}\right\}$, and we have used the fact that $\mathbf{v}_{i} \perp \mathbf{z}_{i}$.

Now consider the following Lyapunov function

$$
w(\mathbf{z})=\frac{n}{2}|\mathbf{p}|^{2}=\frac{1}{2 n} \mathbf{z}^{T} \mathbf{1 1}^{T} \mathbf{z}
$$

which is minimized for the balanced formation. Given the gradient of $w(\mathbf{z})$ :

$$
\frac{\partial w(\mathbf{z})}{\partial \mathbf{z}_{i}}=\frac{1}{n}\left(\mathbf{1 1}^{T} \mathbf{z}\right)_{i}=\frac{1}{n} \mathbf{1}^{T} \mathbf{z}=\mathbf{p}
$$

the time derivative of $w(\mathbf{z})$ becomes

$$
\begin{aligned}
\dot{w}(\mathbf{z}) & =\sum_{i=1}^{n}<\frac{\partial w(\mathbf{z})}{\partial \mathbf{z}_{i}}, \dot{\mathbf{z}}_{i}>=\sum_{i=1}^{n}<\mathbf{p}, \mathbf{v}_{i}>u_{i} \\
& \leq-\frac{n \kappa}{|\mathbf{z}|_{\max }} \sum_{i=1}^{n}<\mathbf{p}, \mathbf{v}_{i}>^{2} \leq 0
\end{aligned}
$$

where we have used (4).

A simple application of LaSalle's invariance principle over the configuration space which is an $n$-torus and therefore compact reveals that all trajectories starting in anywhere on the $n$-torus converge to the largest invariant sets in $E=$ $\{\mathbf{z} \mid \dot{w}(\mathbf{z})=\mathbf{0}\}$. This set is characterized by $\left\langle\mathbf{p}, \mathbf{v}_{i}>=0\right.$, for all $i \in\{1, \ldots, n\}$. Therefore the equilibria are given by either $\mathbf{p}=\mathbf{0}$, or $\mathbf{p} \perp \mathbf{v}_{i}$ for all $i \in\{1, \ldots, n\} \cdot \mathbf{p}=\mathbf{0}$ is the global minimum of $w(\mathbf{z})$ and is asymptotically stable. At the equilibrium we have $u_{i}=0$ for all $i \in\{1, \ldots, n\}$ and as a result the geometric center remains fixed because $\dot{\mathbf{p}}=\sum_{i} u_{i} \mathbf{v}_{i}=\mathbf{0}$.

The critical points given by $\mathbf{p} \perp \mathbf{v}_{i}$ correspond to a set of configurations that $m$ agents are at antipodal position from the 
other $n-m$ agents, where $1 \leq m<n / 2$. The instability of these equilibria is proved by showing that if we perturb the system at those equilibria, the system moves away from them and $w(\mathbf{z})$ will be decreasing.

Remark 3.4: The Laplacian matrix of a complete graph equals to $L_{c}=I_{n}-(1 / n) \mathbf{1}_{n} \mathbf{1}_{n}^{T}$. Thus, one can see that minimizing $w(\mathbf{z})$ in (5) is equivalent to maximizing $\mathbf{z}^{T} \bar{L}_{c} \mathbf{z}$ with $\bar{L}_{c}=L_{c} \otimes I_{2}$. The maximum is achieved when all the agents are evenly spaced around the circle.

\section{Ring Topology}

Next we consider the situation that the connectivity graph has a ring topology. We denote this graph with $\mathbb{G}^{\text {ring }}$. We have the following theorem for the balanced circular formations of a group of mobile agents with ring topology.

Theorem 3.5: Consider a system of $n$ agents with kinematics (2). Suppose the connectivity graph has the ring topology $\mathbb{G}^{\text {ring }}$ and each agent applies the balancing control law (3). Let $\phi_{o}$ be the angle to which the relative headings converge, then if $\phi_{o} \in(\pi / 2,3 \pi / 2)$ the balanced equilibrium is locally exponentially stable.

Proof: Let $L_{r}$ be the Laplacian matrix of a graph with a ring topology, and $\bar{L}_{r}=L_{r} \otimes I_{2}$. Input (3) can be written in terms of the Laplacian of the connectivity graph:

$$
\begin{aligned}
u_{i} & =\kappa \sum_{j \in \mathcal{N}_{i}} \frac{1}{\left|\mathbf{z}_{i j}\right|}<\mathbf{z}_{i}-\mathbf{z}_{j}, \mathbf{v}_{i}> \\
& \geq \frac{\kappa}{|\mathbf{z}|_{\max }} \sum_{j \in \mathcal{N}_{i}}<\mathbf{z}_{i}-\mathbf{z}_{j}, \mathbf{v}_{i}> \\
& =\frac{\kappa}{|\mathbf{z}|_{\max }}<\left(\bar{L}_{r} \mathbf{z}\right)_{i}, \mathbf{v}_{i}>, \quad \kappa>0
\end{aligned}
$$

where $\left(\bar{L}_{r} \mathbf{z}\right)_{i} \in \mathbb{R}^{2}$ is the subvector of $\bar{L}_{r} \mathbf{z}$ associated with the $i^{t h}$ agent. Now consider the function

$$
s(\mathbf{z})=\frac{1}{2} \mathbf{z}^{T} \bar{L}_{r} \mathbf{z}
$$

that is maximized for the balanced formation, and this maximum exists because $s(\mathbf{z})$ is bounded from above. Using the dynamics (2) and input (3) we have that

$$
\begin{aligned}
\dot{s}(\mathbf{z}) & =\sum_{i=1}^{n}<\frac{\partial s(\mathbf{z})}{\partial \mathbf{z}_{i}}, \dot{\mathbf{z}}_{i}>=\sum_{i=1}^{n}<\left(\bar{L}_{r} \mathbf{z}\right)_{i}, \mathbf{v}_{i}>u_{i} \\
& \geq \frac{n}{|\mathbf{z}|_{\max }} \sum_{i=1}^{n}<\left(\bar{L}_{r} \mathbf{z}\right)_{i}, \mathbf{v}_{i}>^{2} \geq 0
\end{aligned}
$$

Thus $s(\mathbf{z})$ monotonically increases along the trajectories of system (2) with input (3), and converges to equilibria corresponding to

$$
<\left(\bar{L}_{r} \mathbf{z}\right)_{i}, \mathbf{v}_{i}>=0, \quad \forall i \in\{1, \ldots, n\} .
$$

Let us characterize the set of equilibria given by (9). We represent the unit vector $\mathbf{z}_{i}$ in the rotating frame by $\mathbf{z}_{i}=$ $\left[\begin{array}{ll}\cos \theta_{i} & \sin \theta_{i}\end{array}\right]^{T}$. Then $\mathbf{v}_{i}=\left[\begin{array}{ll}-\sin \theta_{i} & \cos \theta_{i}\end{array}\right]^{T}$, and (9) is equivalent to

$$
\sum_{j \in \mathcal{N}_{i}} \sin \left(\theta_{i}-\theta_{j}\right)=0, \quad \forall i \in\{1, \ldots, n\} .
$$

Let $\boldsymbol{\theta}=\left[\theta_{1}, \ldots, \theta_{n}\right]^{T}$. Then (10) becomes

$$
B \sin \left(B^{T} \boldsymbol{\theta}\right)=0,
$$

where $B \in \mathbb{R}^{n \times e}$ is the incidence matrix of $\mathbb{G}^{\text {ring }}$, where $e=|\mathcal{E}|$. For $\mathbb{G}^{\text {ring }}, n=e$ and $B$ is a circulant matrix that satisfies $B \mathbf{1}_{e}=\mathbf{0}$. Let $\boldsymbol{\phi}=B^{T} \boldsymbol{\theta}$. Then the equilibria of system (11) are characterized by

$$
\begin{aligned}
\sin \phi & =\alpha \mathbf{1}_{e} \\
\mathbf{1}_{e}^{T} \boldsymbol{\phi} & =m \pi .
\end{aligned}
$$

Vector $\phi$ satisfies equation (12) iff $\phi_{k}=\left\{\phi_{o}, \pi-\phi_{o}\right\}$ for all $k \in\{1, \ldots, e\}$ and $\phi_{o} \in(0,2 \pi)$. Equation (13) is satisfied if $\phi_{o}=(m / e) \pi$ for $m \in \mathbb{N}$.

Next we prove the (local) exponential stability of the relative equilibria, i.e., the balanced state. For the proof of the exponential stability of the equilibrium $\phi=\phi_{o} \mathbf{1}_{e}$ we consider the linearization of system (11) about $\phi_{o}$. The Jacobian of system $\dot{\boldsymbol{\theta}}=\kappa B \sin \left(B^{T} \boldsymbol{\theta}\right)$ at the equilibrium is

$$
J=\kappa B \operatorname{diag}\left(\cos \phi_{o}\right) B^{T}=\kappa \cos \phi_{o} B B^{T}
$$

where $\operatorname{diag}\left(\cos \phi_{o}\right)$ is an $e \times e$ matrix with $\cos \phi_{o}$ as its diagonal elements. Since $\kappa>0$, the linearized system $\dot{\boldsymbol{\theta}}=J \boldsymbol{\theta}$ is exponentially stable if $\phi_{o} \in(\pi / 2,3 \pi / 2)$.

As a result at the equilibrium the final configuration for $\mathbb{G}^{\text {ring }}$ is either a star polygon (for $n$ odd), or a line (for $n$ even) with odd-indexed agents on one side and even-indexed agents on the other side. This can be seen by noting that for a $\{n / d\}$ polygon, the angle between the connected nodes is $2 \pi d / n$. Thus, the stable equilibria given by $\phi_{o} \in(\pi / 2,3 \pi / 2)$ correspond to polygons with $d \in(n / 4,3 n / 4)$.

For example, for $n=5$, the stable polygons are $\{5 / 3\}$ and $\{5 / 4\}$ which are the same polygons with reverse ordering of the nodes. Simulations suggest that the largest region of attraction for $n$ even belongs to a polygon $\{n / d\}$ with $d=$ $n / 2$, and a star polygon $\{n / d\}$ with $d=(n \pm 1) / 2$ for $n$ odd. These results are observed in experiments with real robots as demonstrated in Section V.

\section{Bearing-based control law}

In this section, we write input (3) in terms of a parameter that is measurable using a simple visual system. Similar attempt was done in [13] to obtain vision-based control laws for flocking of a group of nonholonomic agents. Let $\mathbf{r}_{i}=$ $\left[\begin{array}{ll}x_{i} & y_{i}\end{array}\right]^{T}$ be the location of agent $i$ in a fixed world frame, and $\mathbf{v}_{i}=\left[\dot{x}_{i} \dot{y}_{i}\right]^{T}$ be its velocity vector. The heading or orientation of agent $i$ is then given by

$$
\theta_{i}=\operatorname{atan} 2\left(\dot{y}_{i}, \dot{x}_{i}\right)
$$




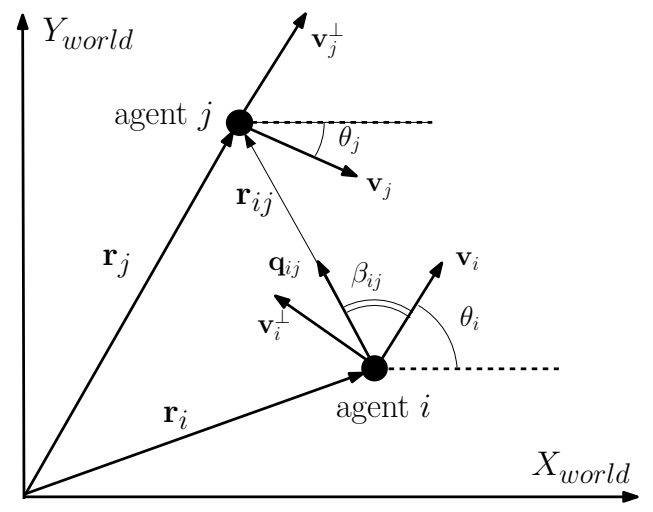

Fig. 3. Bearing angle $\beta_{i j}$ is measured as the angle between the velocity vector (along body $x$-axis) and vector $\mathbf{r}_{i j}$, which connects the two neighboring agents.

Given the above definitions, dynamic model (1) becomes the unicycle model:

$$
\begin{aligned}
\dot{x}_{i} & =v \cos \theta_{i} \\
\dot{y}_{i} & =v \sin \theta_{i} \\
\dot{\theta}_{i} & =\omega_{i}
\end{aligned}
$$

where $\omega_{i}$ is the angular velocity of agent $i$, and $v$ is the constant linear velocity (assuming $v=1$ in this section). Let $\beta_{i j}$ be the relative angle between agents $i$ and $j$ as measured in the local coordinate frame of agent $i$. The bearing angle $\beta_{i j}$ is defined as (see Figure 3):

$$
\beta_{i j} \doteq \operatorname{atan} 2\left(y_{i}-y_{j}, x_{i}-x_{j}\right)-\theta_{i} .
$$

The only visual parameter that is required for generating a balanced circular formation is the bearing angle, $\beta_{i j}$. It is remarkable that we can generate interesting global patterns using only a single measurement of the bearing angle. Note that the inner product of two vectors is independent from the coordinate system in which they are expressed. Thus, given $\mathbf{v}_{i}=\left[\begin{array}{ll}1 & 0\end{array}\right]^{T}$ and $\mathbf{q}_{i j}=\left[\begin{array}{ll}\cos \beta_{i j} & \sin \beta_{i j}\end{array}\right]^{T}$ in the body frame of agent $i$, the control input for a balanced circular formation can be written as:

$$
\omega_{i}=\omega_{o}-\kappa \sum_{j \in \mathcal{N}_{i}}<\mathbf{v}_{i}, \mathbf{q}_{i j}>=\omega_{o}-\kappa \sum_{j \in \mathcal{N}_{i}} \cos \beta_{i j},
$$

where $\kappa>0$. Input (17) is the desired bearing-only control input.

\section{Balanced Circular Formation with Collision AVOIDANCE}

The central contribution of this work is providing a simple bearing-only control law for reaching a balanced circular formation. Of course in reality any formation control requires collision avoidance, and indeed collision avoidance cannot be done without range. What we show here is that the two tasks can be done with decoupled additive terms in the control law, where the term for circular formation depends only on bearing.

To ensure collision avoidance and cohesion of the formation, an inter-agent potential function [14], [15] is defined. A

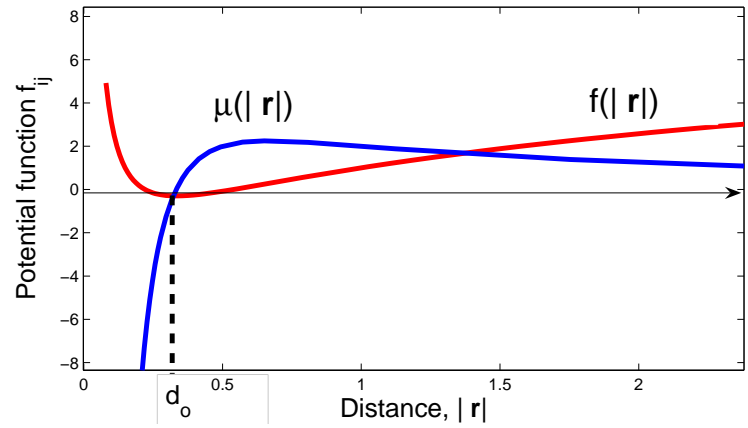

Fig. 4. Artificial potential function $f_{i j}$, and the norm of its gradient $\mu_{i j}$.

control law from this artificial potential function results in simple steering behaviors known as separation and cohesion that govern how each agent maneuvers based on the relative position of its neighbors. The global minimum of this function is where all the agents are at the desired distances.

It was shown in [15] that only if the underlying proximity graph is a spanning tree, the formation stabilizes at a state where the potential function is at the global minimum, and all the agents are at the desired distances. Whereas, in the general case, the multi-agent system reaches a stable state where the potential energy of the system is minimized (a local minimum). Next we formally define the notion of potential function used in this paper.

The potential function $f_{i j}\left(\left|\mathbf{r}_{i j}\right|\right)$ is a symmetric function of the distance $\left|\mathbf{r}_{i j}\right|=l_{i j}$ between agents $i$ and $j$, and is defined as follows [15]:

Definition 4.1 (Potential Function): Potential $f_{i j}$ is a differentiable, nonnegative function of the distance $\left|\mathbf{r}_{i j}\right|$ between agents $i$ and $j$ such that,

- $f_{i j} \rightarrow \infty$ as $\left|\mathbf{r}_{i j}\right| \rightarrow 0$.

- $f_{i j}$ attains its unique minimum when agents $i$ and $j$ are located at a desired distance.

This definition ensures that minimization of the inter-agent potential functions leads to the desired cohesion and separation in the group. Agent $i$ 's total potential is given by

$$
f_{i}=\sum_{j \in \mathcal{N}_{i}} f_{i j}\left(\left|\mathbf{r}_{i j}\right|\right) .
$$

The requirements for $f_{i j}$ given in Definition 4.1 support a large class of functions. Similar potential functions as the following are used in both [15] and [16]:

$$
f_{i j}=\frac{d_{0}}{\left|\mathbf{r}_{i j}\right|}+\log \left|\mathbf{r}_{i j}\right|,
$$

where $d_{0}$ is the desired distance between the pair $(i, j)$. This choice of $f_{i j}$ provides an attractive force when an agent is moving away from the group, and a repulsive force when two agents get too close to each other. The gradient of this function is given by

$\nabla_{\mathbf{r}_{i j}} f_{i j}=\frac{\mathbf{r}_{i j}}{\left|\mathbf{r}_{i j}\right|}\left(\frac{1}{\left|\mathbf{r}_{i j}\right|}-\frac{d_{0}}{\left|\mathbf{r}_{i j}\right|^{2}}\right)=\mu\left(\left|\mathbf{r}_{i j}\right|\right) \mathbf{q}_{i j}=\mu_{i j} \mathbf{q}_{i j}$ 
where $\mathbf{q}_{i j}$ is the unit-length bearing vector between agent $i$ and its neighbor $j$. See Figure 4 for the plots of the potential function $f_{i j}$, and the norm of its gradient $\mu_{i j}=\left|\nabla_{\mathbf{r}_{i j}} f_{i j}\right|$.

The control input for balanced formations must have an additional components $\alpha_{i}$ that controls the spacing between the agents. $\alpha_{i}$ steers the agents to avoid collisions or pull them together if they are separating too far apart. For the inserted force to be gyroscopic, it must be perpendicular to the velocity vector $\mathbf{v}_{i}$ and along $\mathbf{v}_{i}^{\perp}$. The force is proportional to the negative gradient of the potential function $f_{i}$. Thus, as a result the spacing control must have the form

$$
\alpha_{i}=-\kappa_{s}<\mathbf{v}_{i}^{\perp}, \nabla_{\mathbf{r}_{i}} f_{i}>, \quad \kappa_{s}>0 .
$$

Note that since $\mathbf{r}_{i j}=\mathbf{r}_{j}-\mathbf{r}_{i}$ we have

$$
\nabla_{\mathbf{r}_{i}} f_{i}=-\nabla_{\mathbf{r}_{i j}} f_{i}=-\sum_{j \in \mathcal{N}_{i}} \nabla_{\mathbf{r}_{i j}} f_{i j}=-\sum_{j \in \mathcal{N}_{i}} \mu_{i j} \mathbf{q}_{i j} .
$$

Finally, we have the following proposition for reaching the balanced circular formation with collision avoidance:

Proposition 4.2: Consider a system of $n$ agents with $d y$ namics (1) and applying the control input

$$
\begin{aligned}
\omega_{i} & =\omega_{o}+u_{i}+\alpha_{i} \\
& =\omega_{o}-\kappa_{b} \sum_{j \in \mathcal{N}_{i}}<\mathbf{v}_{i}, \mathbf{q}_{i j}>+\kappa_{s} \sum_{j \in \mathcal{N}_{i}} \mu_{i j}<\mathbf{v}_{i}^{\perp}, \mathbf{q}_{i j}>
\end{aligned}
$$

where $\kappa_{b}>0$ and $\kappa_{s}>0$. Given that $\mathbb{G}$ remains connected, the $n$-agent system asymptotically reaches the balanced formation, and collisions between the interconnected agents are avoided.

\section{EXPERIMENTS}

In this section we show the results of experimental tests for two important cases: (a) the complete-graph topology and (b) the ring topology. But first, let us describe the experimental testbed.

\section{A. Experimental Testbed Components}

The experimental testbed consists of many components that are interfaced together to create the total system. In the discussion that follows, we present the robots, software and infrastructure of the testbed.
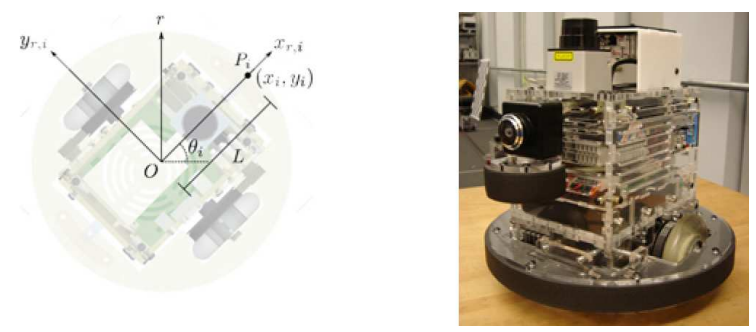

Fig. 5. Scarab is a small robot with a differential drive axil. LED markers are placed on top of each Scarab for tracking and ground-truth verification.
Robots: We use a series of small form-factor robots called Scarab [17]. The Scarab is a $20 \times 13.5 \times 22.2 \mathrm{~cm}^{3}$ indoor ground platform with a mass of $8 \mathrm{~kg}$. Each $S c a r a b$ is equipped with a differential drive axle placed at the center of the length of the robot with a $21 \mathrm{~cm}$ wheel base (See Figure 5). Each Scarab is equipped with an onboard computer, power management system and wireless communication. Each robot is actuated by stepper motors that allows us to model it as a point robot with unicycle kinematics (15) for its velocity range. The linear velocity of each robot is bounded at $0.2 \mathrm{~m} / \mathrm{s}$. Each robot is able to rotate about its center of mass at speeds below $1.5 \mathrm{rad} / \mathrm{s}$. Typical angular velocities resulting from the control law were below $0.5 \mathrm{rad} / \mathrm{s}$.

Software: Every robot is running identical modularized software with well defined interfaces connecting modules via the Player robot architecture system [18], which consists of libraries that provide access to communication and interface functionality. The Player also provides a close collaboration with the three-dimensional physics-based simulation environment Gazebo. Gazebo provides the powerful ability to transition transparently from code running on simulated hardware to real hardware.

Infrastructure: In the experiments, visibility of the robot's set of neighbors is the main issue. Using omnidirectional cameras seems to be a natural solution. However, in order to reduce the on-board computation, a tracking system consisting of LED markers on the robots and eight overhead cameras is designed. This ground-truth verification system can locate and track the robots with position error of approximately $2 \mathrm{~cm}$ and an orientation error of $5^{\circ}$. The overhead tracking system allows control algorithms to assume pose is known in a global reference frame. The process and measurement models fuse local odometry information and tracking information from the camera system. Each robot locally estimates its pose based on the globally available tracking system data and local motion, using an extended Kalman filter. We process global overhead tracking information but hide the global state of the system from each robot, providing only the current state of the robot as well as the positions of each robot's set of neighbors. In this way, we use the tracking system in lieu of an inter-robot sensor implementation.

In all the experiments the neighborhood relations, i.e., the connectivity graphs, are fixed and undirected. Each robot computes the bearing angles with respect to its neighbors from equation (16), and applies the vision-based control input (17). The conclusions for each set of experiments are drawn from significant number of successful trials that supported the effectiveness of the designed controller. The results of the experiments are provided in the following subsections.

\section{B. Complete-Graph Topology}

First we applied the bearing-only control law (17) to a group of $n=5$ robots without considering collision avoidance among the agents. In Figures 6 (a) through 6 (d) snapshots from the actual experiment are shown, and in Figures 6 (e) 


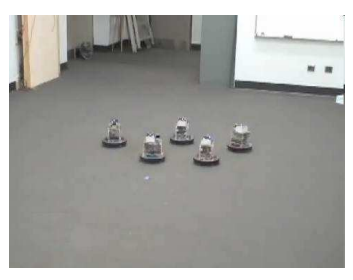

(a)

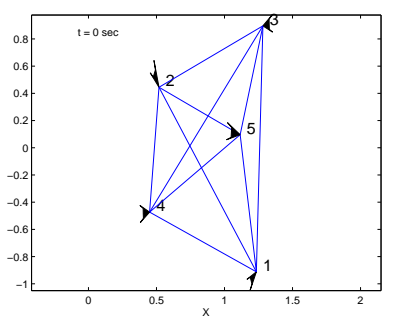

(e)

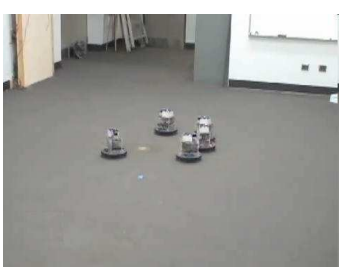

(b)

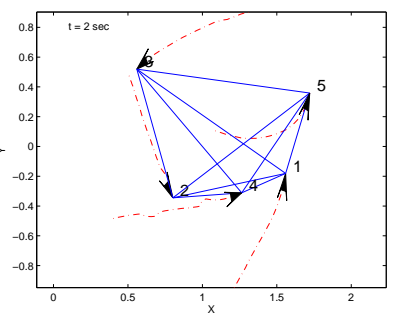

(f)

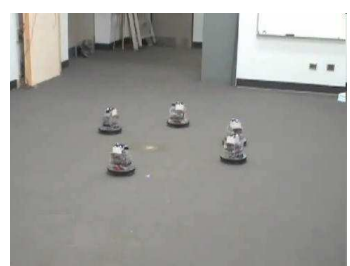

(c)

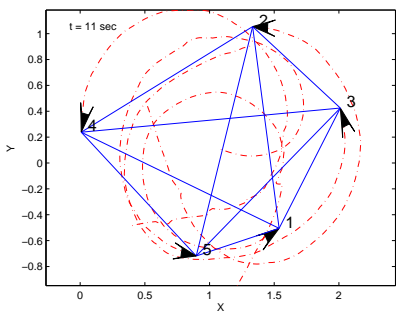

(g)

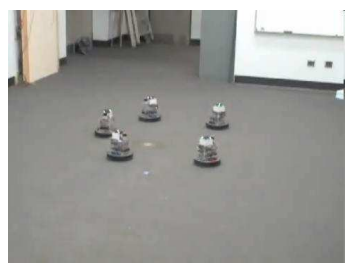

(d)

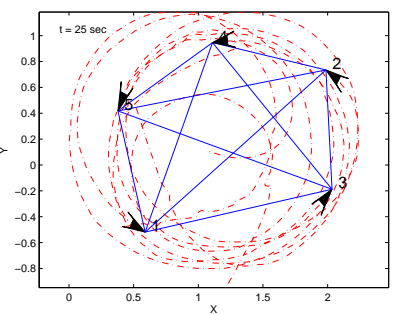

(h)

Fig. 6. Five Scarabs form a circular formation starting with a complete-graph topology. (a) At time $t=0$ robots starts at random positions and orientations. (b) $t=2 \mathrm{sec}$. (c) $t=11 \mathrm{sec}$. (d) At $t=25 \mathrm{sec}$. the robots reach a stable balanced configuration around a circle with radius of $1 \mathrm{~m}$. Figures (e) through (h) show the actual trajectories of the robots and their connectivity graph at the times specified above. Figure 6(h) shows that the final configuration is a regular polygon.

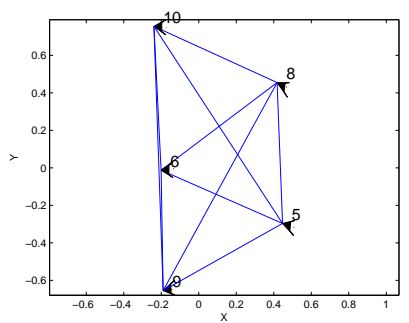

(a)

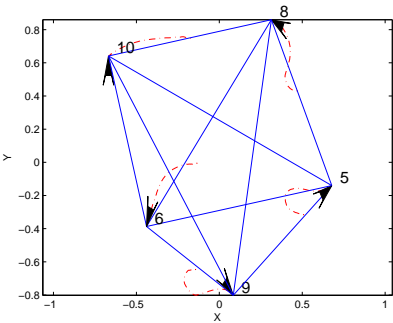

(b)

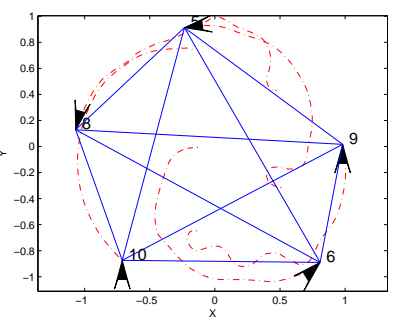

(c)

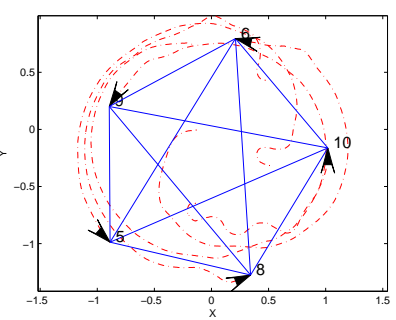

(d)

Fig. 8. Five Scarabs form a circular formation starting with a complete-graph topology while avoiding collisions. (a) $t=0$ sec. (b) $t=8$ sec. (c) $t=20$ sec. (d) At $t=36 \mathrm{sec}$. the robots reach a stable balanced configuration around a circle with radius of $1 \mathrm{~m}$. Figures (a) through (d) show the actual trajectories of the robots and their connectivity graph at the times specified above.

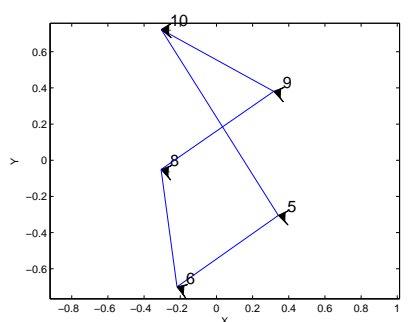

(a)

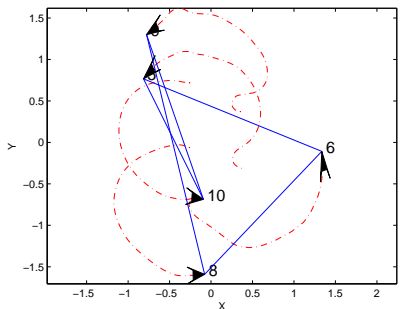

(b)

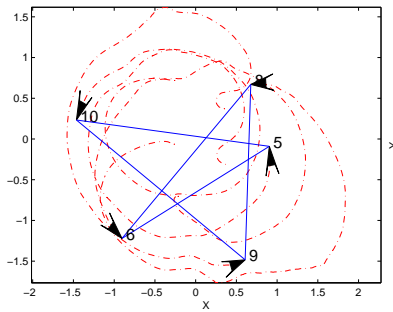

(c)

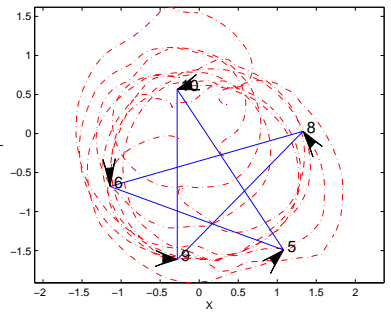

(d)

Fig. 9. Five Scarabs form a circular formation starting with a ring topology while avoiding collisions. (a) $t=0$ sec. (b) $t=16$ sec. (c) $t=40$ sec. (d) At $t=80 \mathrm{sec}$. the robots reach a stable balanced configuration, which is the star polygon $\{5 / 3\}$, around a circle with radius of $1 \mathrm{~m}$. Figures (a) through (d) show the actual trajectories of the robots and their connectivity graph at the times specified above.

through $6(\mathrm{~h})$ the corresponding trajectories, generated from overhead tracking information, are demonstrated. Note that for the complete-graph topology the ordering of the robots in the final configuration is not unique, and it depends on the initial positions.
Since there was no collision avoidance implemented in the experiments of Figure 6 the robots could become undesirably close to one another as it can be seen in Fig. 6 (b). However, by applying control input (21) no collisions occur among the robots as they reach the equilibrium. The actual trajectories 


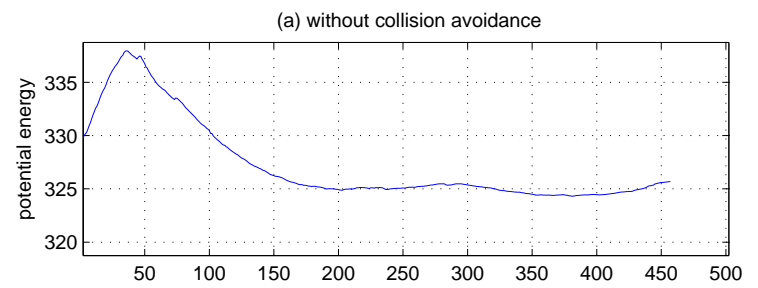

(b) With collision avoidance

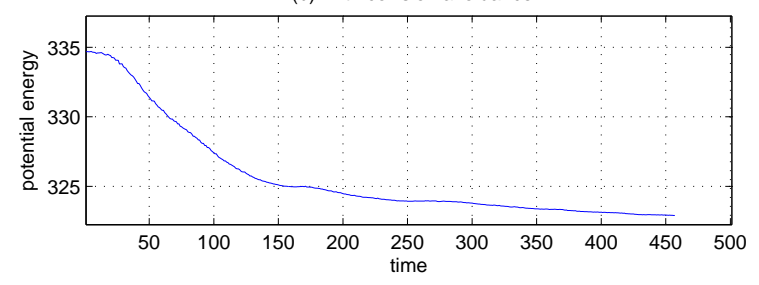

Fig. 7. Comparison of the values of the 5-agent system's potential energy while robots are applying (a) control input (17) and (b) control input (21) with collision avoidance.

of $n=5$ robots for this scenario are shown in Figure 8 . The comparison of the potential energies of the system with and without $\alpha_{i}$ term (20) are presented in Figure 7. The potential energy of the system is computed from $f=\sum_{i=1}^{n} f_{i}$ where $f_{i}$ is given by (18). The peak in Fig. 7 (a) corresponds to the configuration observed in Fig. 6 (b) where robots become too close to each other. By using the control input (21) the potential energy of the 5 -agent system monotonically decreases (see Fig. 7 (b)) and the system stabilizes on a state that the potential energy of the entire system is minimized.

\section{Ring Topology}

If every robot can only "sense" two other robots in the group, the topology of the connectivity graph will be a ring topology. Since the connectivity graph is assumed fixed, the agents need to be numbered during the experiments. For $n$ even, the largest region of attraction is an $\{n / d\}$ polygon with $d=n / 2$, which is not physically possible, because it requires that robots with even indices to stay on one side of a line segment and robots with odd indices to stay at the other side. For $n$ odd, both simulations and experiment suggest that the largest region of attraction belong to star polygon $\{n / d\}$ with $d=(n \pm 1) / 2$, therefore, there are only two possible ordering of the robots in the final circular formation. Figure 9 shows that in our experiment the robots are stabilized to the star polygon $\{5 / 3\}$.

Remark 5.1: When the communication graph is a fixed, directed graph with a ring topology, where agent $i$ could only see agent $(i+1) / \bmod (n)$, then the $n$-agent system would behave like a team of robots in cyclic pursuit [9].

\section{Conclusions}

We developed a control input for balanced circular formations of a group of ground robots that required only the measurements of the bearing angles with respect to the set of neighbors. Since the bearing angles could be simply measured using basic visual sensors on a robot, this control input could be considered a vision-based input. The results show how we can generate interesting global patterns using only local information, and without knowing a global reference frame. To improve the experimental results, we added collision avoidance capabilities to our control input for balanced formations.

In future we would like to implement the proposed control algorithm on robots with vision sensors. If the robot-mounted visual sensor for bearing measurements is a camera with a limited field of view, the underlying connectivity graph will be a directed graph. The study of circular formations with a directed graph is an ongoing work.

\section{REFERENCES}

[1] A. Okubo, "Dynamical aspects of animal grouping: swarms, schools, flocks, and herds," Advances in Biophysics, vol. 22, pp. 1-94, 1986.

[2] D. J. Low, "Following the crowd," Nature, vol. 407, pp. 465-466, 2000.

[3] I. Couzin, J. Krause, R. James, G. Ruxton, and N. Franks, "Collective memory and spatial sorting in animal groups," Journal of Theoretical Biology, vol. 218(1), p. 111, 2002.

[4] H. Levine, E. Ben-Jacob, I. Cohen, and W.-J. Rappel, "Swarming patterns in microorganisms: Some new modeling results," in IEEE Conference on Decision and Control, December 2006, pp. 5073 - 5077.

[5] D. A. Paley, N. E. Leonard, R. Sepulchre, D. Grnbaum, and J. K. Parrish, "Oscillator models and collective motion," IEEE Control Systems Magazine, vol. 27, no. 4, pp. 89 - 105, August 2007.

[6] R. Sepulchre, D. Paley, and N. Leonard, "Stabilization of planar collective motion: All-to-all communication," IEEE Transaction of Automatic Control, vol. 52, no. 5, pp. 811 - 824, May 2007.

[7] N. Moshtagh and A. Jadbabaie, "Distributed geodesic control laws for flocking of nonholonomic agents," IEEE Transaction on Automatic Control, vol. 52, April 2007.

[8] J. Marshall, M. Broucke, and B. Francis, "Formations of vehicles in cyclic pursuit," IEEE Transaction of Automatic Control, November 2004.

[9] J. Marshall, T. Fung, M. Broucke, G. D'Eleuterio, and B. Francis, "Experiments in multirobot coordination," Robotics and Autonomous Systems, vol. 54, pp. 265-275, November 2006.

[10] M. Pavone and E. Frazzoli, "Decentralized policies for geometric pattern formation and path coverage," ASME Journal on Dynamic Systems, Measurement, and Control, 2007.

[11] J. Jeanne, N. Leonard, and D. Paley, "Colletive motion of ring-coupled planar particles," in 44th IEEE Conference on Decision and Control, Seville, Spain, December 2005, pp. 3929 - 3934

[12] E. Justh and P. Krishnaprasad, "Equilibria and steering laws for planar formations," Systems and Control letters, vol. 52, no. 1, pp. 25-38, May 2004.

[13] N. Moshtagh, A. Jadbabaie, and K. Daniilidis, "Vision-based control laws for distributed flocking of nonholonomic agents," in IEEE International Conference of Robotics and Automation, Orlando, Florida, May 2006, pp. $2769-2774$

[14] P. Ogren, E. Fiorelli, and N. Leonard, "Cooperative control of mobile sensing networks: Adaptive gradient climbing in a distributed environment," IEEE Transaction of Automatic Control, vol. 49(8), pp. 1292 1302, August 2004.

[15] H. Tanner, A. Jadbabaie, and G. Pappas, "Flocking in fixed and switching newtorks," IEEE Transaction of Automatic Control, vol. 52 , pp. 863-868, 2007.

[16] E. Justh and P. Krishnaprasad, "Natural frames and interacting particles in three dimensions," in IEEE Conference on Decision and Control, December 2005, pp. $2841-2846$.

[17] N. Michael, J. Fink, and V. Kumar, "Controlling a team of ground robots via an aerial robot," in Proc. of the IEEE/RSJ Int. Conf. on Intelligent Robots and Systems, San Diego, CA, Nov. 2007.

[18] B. Gerkey, R. Vaughan, and A. Howard, "The player/stage project: Tools for multi-robot and distributed sensor systems," in Proc. of the Int. Conf. on Advanced Robotics, Portugal, June 2003, pp. 317-323. 